\title{
Modified Recovery Algorithms Using proposed algorithm for Compressive Sampling
}

\author{
Wafaa A. Shalaby, Waleed Saad, Mona Shokair and Moawad I. Dessouky \\ Faculty of Electronic Engineering, Menoufia University, Menouf 32952, Egypt
}

(Received: 31-March-2016 - Accepted: 20-May-2016)

\section{Abstract}

Compressive sampling (CS) has been an effective research area which plays an efficient role in many applications such as cognitive radio, imaging, radar and many other applications. In CS only a small number of linear measurements are used for reconstruction of the signal. The significant condition for dealing with compressed sensing system is that the signal in the input must be sparse. Most signals in nature are sparse or can be transformed to sparse by using any transform domain. This paper modifies all the recovery algorithms by using the proposed complex to real transformation algorithm. Conversion from not sparse signal to sparse by using Fourier transform will produce complexity, where this complexity can be removed using complex to real transformation algorithm and then applying it on all recovery algorithms to enhance their performance. By using the proposed algorithm, the sparse signal will be recovered in minimum error and less time. Also, the signal to error ratio from the recovery process is increased.

\section{Introduction}

Compressive sampling (CS) has a new life and spreads rapidly from 2006 until now. CS had been used in many applications such as Rader, Remote sensing, cognitive radio and other applications. CS uses smaller rate than the Nyquist sampling rate defined by the Nyquist theorem, where the signal must be sampled at least twice the maximum frequency of the signal and then compressed the product of the sampling. CS combines two 
stages of sampling and compression of the signal in one stage called compressed sensing as shown in Fig.1.

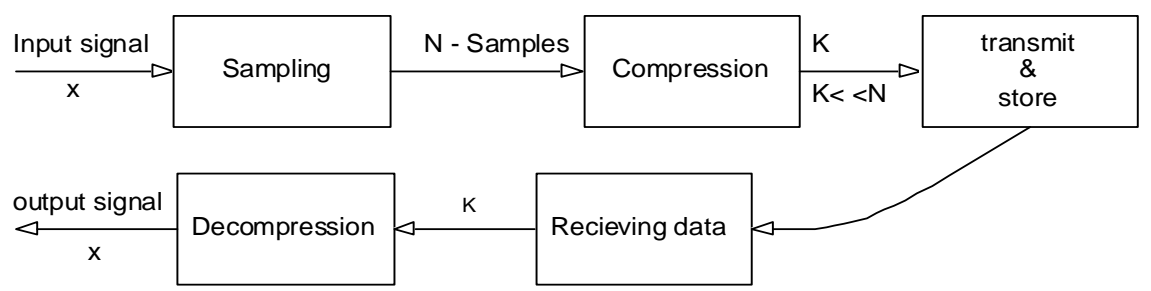

(a)

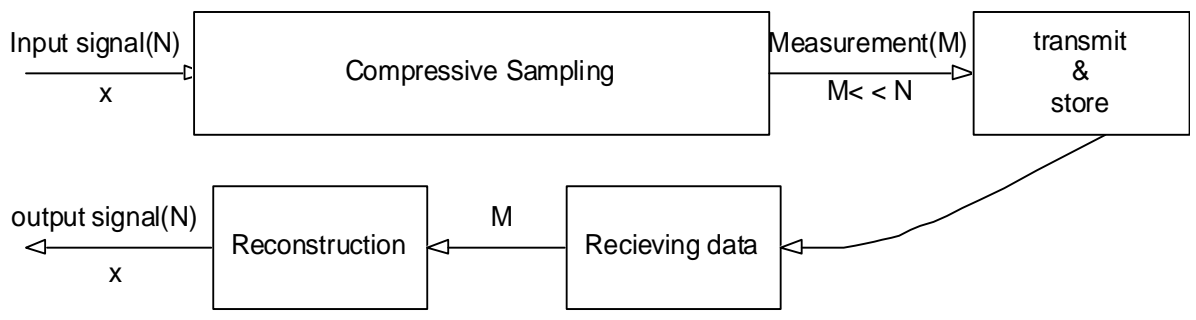

(b)

Fig.1 a) Conventional system, b) Compressed sensing system

* Some of parts of this paper has been taken from [13]

One of the main conditions for CS is the signal sparsity, where the signal must be represented as a sparse signal by using any transform domains such as DCT, DFT and DWT [1]. The important aim for using CS is that the sparse signal is sampled and reconstructed by using less number of measurements $(m)$ that represent the signal of length $(n)$ where $(m<<n)$. These linear measurements are achieved by projecting into various spaces that called the measurement space with which these measurements are achieved by using random matrix such as (Gaussian or Bernoulli) that achieves certain properties such as the RIP and NSP [2] to obtain the correct recovery. The main problem in the compressed sensing system is to recover the sparse signal from a small number of measurements. Therefore, different optimization techniques are used to obtainthe optimal solution such as convex optimization or L1-norm methods [3] that depends on linear programming to recover the sparse signal with high guarantee but it takes a long time to approach the solution [4]. Greedy algorithms depend on iteration to reach to the optimal sparse solution in minimum time than convex optimization such as Orthogonal Matching 
Pursuit OMP [5] which has less guarantee than L1-minimization but with faster time to reach to the solution. Compressive sampling Matching Pursuit COsaMP [6] was proposed to decrease the error in OMP to benear from the convex optimization with less time similar to OMP. Hard Thresholding algorithms such as Iterative Hard Thresholding IHT based on negative gradient descent with fixed step size and the Normalized Iterative Hard Thresholding NIHT that varies the step size to enhance the convergence speed [7]. Hard Thresholding Pursuit HTP is the mixed algorithm that combines between two algorithms Iterative Hard Thresholding and Compressive Sampling Matching Pursuit in [8].

In this paper, input complex sinusoids are used for compressive sampling system where removing the complexity that produced during converting the signal to sparse by using the proposed algorithm called complex to real transformation algorithm. The proposed algorithm will expand the measurement matrix from $\left(m^{*} n\right)$ to $\left(m^{*} 2 n\right)$. This can be obtained by putting the real part in the first columns from 1: $\mathrm{n}$ and then assigning the imaginary parts with a negative sign in the next columns from $n+1: 2 n$. Moreover, expanding the complex sparse signal to be $\left(2 n^{*} 1\right)$ instead of $\left(n^{*} 1\right)$ by using the real parts in the first rows and then imaginary parts in the next rows. The real sensing matrix and sparse signal that achieved by using this transformation will contribute to introducing an improvement in reducing error and reaching to the optimum solution in faster time by using the minimum number of iterations. Moreover, the performance of the different optimization algorithms will be enhanced as will show in the simulation analysis.

This paper is organized as follows: Section 2 illustrates the basic concepts of compressed sensing. The basic model of the proposed algorithm will be investigated in Section 3. The mathematical analysis of the proposed algorithm for compressive sensing will be described in Section 4.The modified recovery algorithms by using the proposed algorithm will be introduced in section 5. Simulation results will be discussed in Section 6 . Finally, conclusions will be done in Section 7

\section{The Basic Concepts of Compressed Sensing}

The aim of CS is to identify the location and the values of a sparse signal from a minimum number of measurements. CS system can be described as 
an underdetermined linear system for $(\mathrm{m}<<\mathrm{n})$ which can be represented by [9].

$$
y=A x
$$

CS contains the following parts:

- Representation of the signal $(x)$ in sparse domain.

- Selecting the measurement matrix $(A)$.

- Formation the measurement vector $(y)$.

\section{A. Representation of the signal $(x)$ in sparse domain:}

The sparse signal has a small number of larger elements and other elements are equal to zero and can be defined by using the zero norm $\|x\|_{0}$ as in the following Equation for input signal vector $x=x_{i}$ for $i=\{1,2, \ldots, N\}[10]$.

$$
\|x\|_{0}=\left\{i: x_{i} \neq 0\right\}
$$

Furthermore, a signal is called compressible signal if it includes a small number of non-zero elements and many elements that are small closed to zero. Therefore, the signal that is not completely sparse or compressible signal can be transformed to sparse(s) by using any transform domain $\beta$. Where $x$ is identified as a function of $\beta$ as follows:

$$
x=\beta s
$$

\section{B. The Measurement Matrix}

The measurement matrix (A) of length $(m * n)$ can be called dictionary matrix where each column in it is called anatom. The measurement matrix is divided into three types by the following [11],

1) Random matrices (Bernoulli orGaussian).

2) Orthogonal transform matrices binary or not binary such as (partial Noiselet matrix or scrambled Fourier matrix).

3) Sparse matrices.

The measurement matrix must be contained the following conditions to obtain the optimal solution: 
- Small mutual coherence between the basis $\beta$ and the measurement matrix $A$ in which the correlations between two basis are described by [12],

$$
\mu(A, \beta)=\sqrt{n} \max _{1 \leq i \leq m, 1 \leq j \leq n} \mid\left\langle A_{i}, \beta j\right|
$$

Where, $\mu(A, \beta) \in[1, \sqrt{n}]$

- The condition for restricted isometry property (RIP) is described as follow [9],

$$
\left(1-\delta_{k}\right)\|s\|_{2}^{2} \leq\|A s\|_{2}^{2} \leq\left(1+\delta_{k}\right)\|s\|_{2}^{2}
$$

The condition for achieving the RIP of order $\mathrm{k}$ is that $\delta_{k}$ must be not too closed to one. Actually, achieving this property is very hard. Therefore, choosing random matrices that achieve this properties such as (Bernoulli or Gaussian) matrices.

\section{The measurement vector $(y)$}

In compressed sensing system the number of measurements $(m)$ must be smaller than the length of the signal $(n)$. Selecting the number of measurements is dependent on the mutual coherencebetween the two basis, the measurement vector and the sparsity basis $\mu(A, \beta)$ as in the following equation[12],

$$
m \geq c \cdot \mu^{2}(A, \beta) \cdot k \cdot \log (n)
$$

For the smallest the coherence, few number of samples are required. If $\mu(A, \beta)$ is equal or close to one,the number of samples will be equal to $k \cdot \log (n)$ samples.

The measurement vector related with the sparse signal (s) is given by,

$$
y=A x=A \beta s
$$

Where, $y=\left[y_{1}, y_{2}, \ldots . ., y_{m}\right]^{T} \in R^{n}, A \in R^{m x n}, \quad \beta=\left[\beta_{1}, \beta_{2}, \ldots . ., \beta_{n}\right] \in R^{n x n}$, and $x=\sum_{i=1}^{n} s_{i} \beta_{i}$. 


\section{The Basic Model of the Proposed Algorithm}

The block diagram of a conventional compressed sensing system will be shown in Fig. 2 with adding the block of complex to real before the recovery process in order to remove the imaginary parts in the sensing matrix and in the sparse signal to become real and remove complexity.

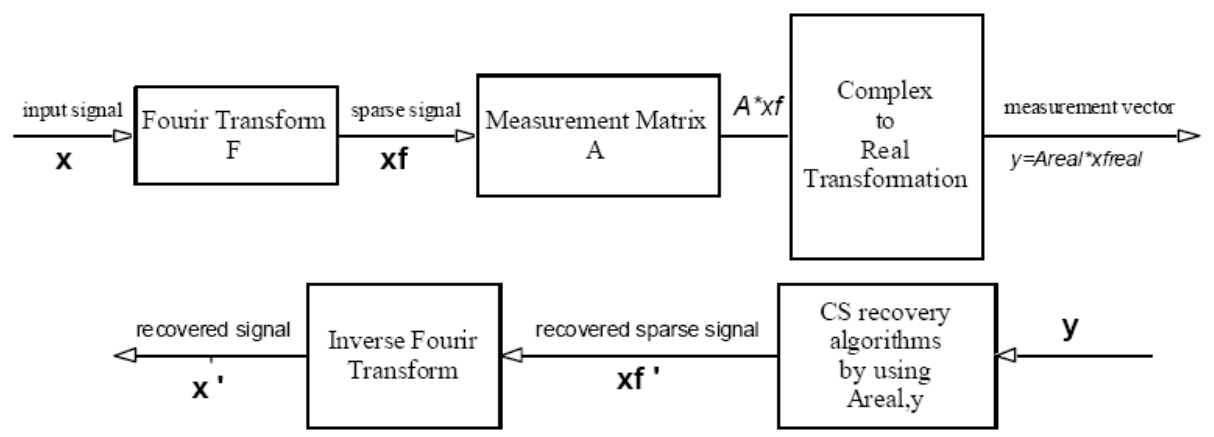

Fig.2: Proposed Compressed Sensing Model [13]

It consists of two parts: sampling process and reconstruction process.

\section{A. Sampling Process}

The input of sinusoid signal ( $x$ ) of length $n$ is converted to the frequency domain to be sparse by using Fourier transform $(F)$ to form a sparse signal $\left(x_{f}\right)$ where

$$
\begin{aligned}
& x_{f}=F x \\
& x=F^{-1} x_{f}
\end{aligned}
$$

The measurement vector (y) are formed by using linear random combination of the signal $\mathrm{x}$ as follows:

$\mathrm{y}=\mathrm{Ax}=\mathrm{A} F^{-1} x_{f}=A^{c s} x_{f}(10)$

The new block that introduced to remove the complexity in both $A^{c s}$, $x_{f}$ to achieve the real matrix $A_{\text {real }}^{c s}$ and real vector $x_{\text {freal }}$ by using the transformation that described in the next section. The measurement vector will become,

$$
\mathrm{y}=A_{\text {real }}^{c s} x_{\text {freal }}
$$




\section{B. Reconstruction Process}

By using the measurement $\mathrm{y}$ and the real sensing matrix $A_{\text {real }}^{c s}$ and any optimization algorithm to obtain a sparse solution $\hat{x}_{f}$ and then using inverse Fourier transform to inverse the sparsity and output the original signal $\hat{x}$.

\section{Mathematical Analysis of the Proposed Algorithm for Compressive Sensing}

Proving that transformation from complex to real will give the same measurement vector y can be shown in [13]. The parameters that used will be defined in Table1.

Table1: Parameters Description [13]

\begin{tabular}{|c|l|}
\hline$A^{c s}$ & $\begin{array}{l}\text { The dictionary matrix is multiplication of the sensing matrix A } \\
\text { and transform matrix } F^{-1}\end{array}$ \\
\hline$F^{-1}$ & Inverse Fourier transform \\
\hline$x_{f}$ & Sparse signal after Fourier transformation \\
\hline$\psi_{m n}$ & The real part of the matrix $A^{c s}$ of the rowmandthe column $n$ \\
\hline$\theta_{m n}$ & The imaginary part of the matrix $A^{c s}$ of the rowmandthe columnn \\
\hline$R_{n}$ & The real part of the sparse signal $x_{f}$ at the row $n$ \\
\hline$I_{n}$ & The imaginary part of the sparse signal $x_{f}$ at the row $n$ \\
\hline$A_{\text {real }}^{c s}$ & The dictionary matrix $A^{c s}$ after the complex to real transformation \\
\hline$x_{f r e a l}$ & The sparse signal $x_{f}$ after the complex to real transformation \\
\hline
\end{tabular}

The recovery process dependson both of $A^{c s}$ and $x_{f}$. Therefore, converting the complexity of both CS measurement matrix and the sparse signal from complex to real will improve recovery output. Assume the complex matrix $A^{c s}$ of dimension $\left(m^{*} n\right)$, the sparse signal $x_{f}$ of length $(n * 1)$ and themeasurement $y$ of length $(m * 1)$ which is defined by, 


$$
\begin{aligned}
& A^{c s}=A^{*} F^{-1}=\left(\begin{array}{ccc}
\psi_{11}+j \theta_{11} & \ldots & \psi_{1 n}+j \theta_{1 n} \\
\vdots & \ddots & \vdots \\
\psi_{m 1}+j \theta_{m 1} & \cdots & \psi_{m n}+j \theta_{m 1}
\end{array}\right) \\
& x_{f}=\left(\begin{array}{lll}
R_{1}+j I_{1} & \ldots \ldots \ldots & R_{n}+j I_{n}
\end{array}\right)^{T}
\end{aligned}
$$

Converting the complex matrix $A^{c s}$ of length $\left(m^{*} n\right)$ to the real matrix $A_{\text {real }}^{c s}$ of dimension $(m * 2 n)$. Then converting the complex sparse signalafter Fourier transform $x_{f}$ of length $\left(n^{*} 1\right)$ to thereal sparse $\operatorname{signal} x_{\text {freal }}$ of length $\left(2 n^{*} 1\right)$, by using the following equations:

$$
\begin{gathered}
A_{\text {real }}^{c s}=\left(\begin{array}{llllll}
\psi_{11} & \cdots & \psi_{1 n} & -\theta_{11} & \cdots & -\theta_{1 n} \\
\vdots & \vdots & \vdots & \vdots & \vdots & \vdots \\
\psi_{m 1} & \cdots & \psi_{m n} & -\theta_{m 1} & \cdots & -\theta_{m n}
\end{array}\right) \\
x_{\text {freal }}=\left(\begin{array}{llllll}
R_{1} & \cdots & R_{n} & I_{1} & \cdots & I_{n}
\end{array}\right)^{T} \\
\mathrm{y}=A^{c s} * x_{f}=A_{\text {real }}^{c s} * x_{\text {freal }}=\left(\begin{array}{c}
\psi_{11} R_{1}+\cdots+\psi_{1 n} R_{n}-\theta_{11} I_{1}-\cdots-\theta_{1 n} I_{n} \\
\vdots \\
\psi_{m 1} R_{1}+\cdots+\psi_{m n} R_{n}-\theta_{m 1} I_{1}-\cdots-\theta_{m n} I_{n}
\end{array}\right)
\end{gathered}
$$

Equation (16) proves thatthe transformation is mathematically correct and output the same measurement vector $y$. This transformation from complex to real beefficient, which providea higher reduction in error becauseof dealing with the real matrix is very easyto use and saving the time as will be shown in the simulation analysis.

\section{Modified Recovery Algorithms by Using the Proposed Algorithm}

The main part of CS system is recovery of the sparse signal(s) from a few measurements (y) by using different optimization techniques. Different types of algorithms typically used for sparse recovery can be summarized asshown in Fig.3. 


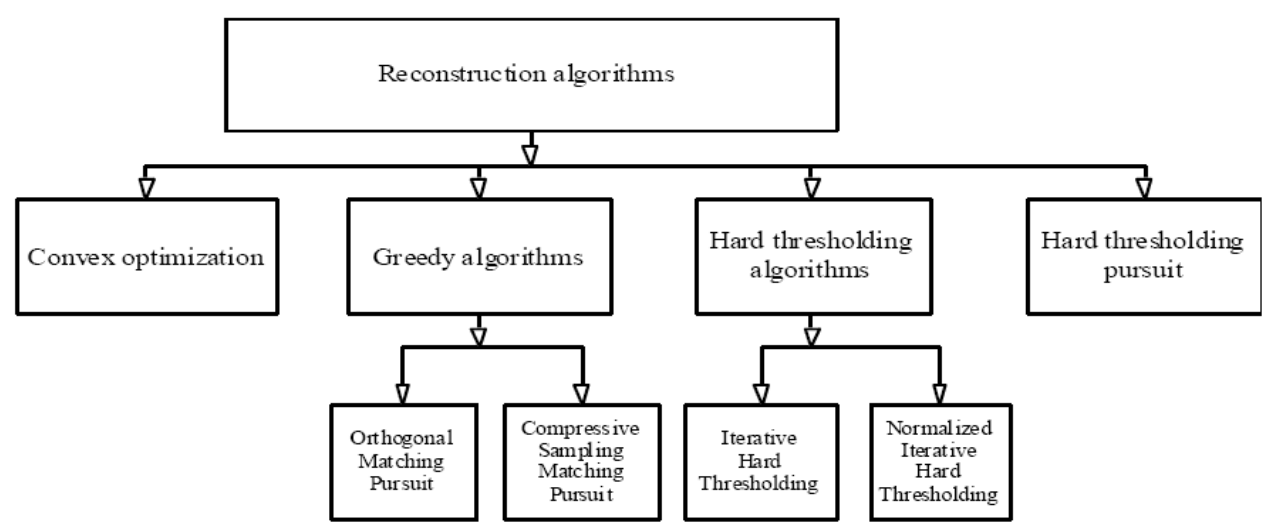

Fig.3: Different Recovery Algorithms

A. Convex Optimization or Basis Pursuit (BP): called L1-norm which is defined by,

$$
\min _{x}\|x\|_{1} \text { s.t } y=A_{\text {real }}^{c s} x
$$

It seeks to find the value of $\hat{x}$ that intersects with $y=A_{\text {real }}^{c s} x$ to produce minimizes $\|x-\hat{x}\|_{1}$. It has advantages that provides strong guarantees and stability for recovery the sparse signal from compressible signals and alsonoisy signals but, it needs long time to reach to the optimal solution. It used linear programming for solution as described in [4] but only with replacing the complex sensing matrix with real one that defined in the proposed algorithm to improve the performance of the recovery process as will discuss in the simulation.

B. Greedy Algorithms: such as COSAMP, which is quite fast in time that is required to obtain the optimal solution but has small guarantees than BP provides. It depends on selecting $2 \mathrm{~s}$ atom per each iteration in which this algorithm expands the support set by $2 \mathrm{~s}$ largest elements in each iteration. COSAMP can be reconstructed the original signal as in [6]: At the beginning, assume that initial solution $x^{0}=0$ and residual $(\mathrm{r})=\mathrm{y}$.Iteration starts atk $=0$. Support $(T)=\varnothing$. In each step, 2 s atoms of $A$ is correlated with the residual of measurements $(\mathrm{y}-\mathrm{Ax})$ and its index is then addedto the support. Then, solve the least square minimization through the current support estimated. Then update the residual $\mathrm{r}$, until reaching to $\hat{x}^{k+1}=\hat{x}^{k}$. 
Then the output becomes $\hat{x}^{k}$. The modified COSAMP algorithm after applying the proposed technique by replacing each complex sensing matrix with real matrix can be summarized as follows:

The proposed COSAMP algorithm

Input: $y, A_{\text {real }}^{c s}, s$
Initialize: $k=0, x^{0}=0, r^{0}=y, \mathrm{~T}=\varnothing$
Steps:
For $k=1,2, \ldots \ldots$
$T^{k}=T^{k-1} \cup \operatorname{supp}\left(\mathrm{H}_{2 s}\left(A_{\text {real }}^{T} r^{k-1}\right)\right.$
$x_{T}^{k}=\underset{\tilde{x}_{T}^{k}}{\operatorname{argmin}}\left\|y-A_{T^{k}} \tilde{x}_{T^{k}}\right\|_{2}^{2}$
$r^{k}=y-A_{\text {real }}^{c s} x^{k}$
End if $\left(\left\|r^{k}\right\|_{2} \leq E P S\right)$
Output: $\boldsymbol{x}=x^{k}$

\section{Hard Thresholding Algorithms:}

IHT is an example on hard thresholding algorithms which it is a simple algorithm that depends on negative gradient descend method [7]. It is an iterative algorithm which the sparse signal can be recovered with more accuracy and in minimum run time than BP.The modified IHT algorithm can be summarized by,

The proposed IHT algorithm

Input: $y, A_{\text {real }}^{c s}, s$

Initialize: $K=0, x^{0}=0, r^{0}=y$

Steps:

For $k=1,2, \ldots \ldots$

$x^{k}=H_{s}\left(x^{k-1}+\mu A_{\text {real }}^{T}\left(\mathrm{y}-A_{\text {real }}^{c s} x^{k-1}\right)\right)$

$r^{k}=y-A_{\text {real }}^{c s} x^{k}$

End for if $\left(\left\|r^{k}\right\|_{2} \leq\right.$ EPS $)$

Output: $\boldsymbol{x}=x^{k}$ 
$H_{s}$ is a hard thresholding operator that puts all the indicies below $\mathrm{s}$ to zero, ${ }^{\mu}$ is the step size parameter that varies linearly to reach a linear rate of convergence and avoids instability in the algorithm

\section{Hard Thresholding Pursuit HTP:}

HTP algorithm combines between the two previous algorithms IHT and COSAMP which introduces a minimization in the time for reaching the optimal solution and also exceedssignal to error ratio [8]. HTP algorithm uses negative gradient descent for estimating the support $\mathrm{T}$ such as IHT algorithm and then solving the least square minimization through the current support estimated T. The modified HTP algorithm after applying the proposed algorithm can be summarized as follows:

The proposed HTP algorithm

Input: $y, A_{\text {real }}^{c s}, s$
Initialize: $k=0, x^{0}=0, r^{0}=y, \mathrm{~T}=\varnothing$
Steps:
For $k=1,2, \ldots \ldots$
$x^{k}=\left(x^{k-1}+\mu A_{\text {real }}^{T}\left(\mathrm{y}-A x^{k-1}\right)\right)$
$T=\operatorname{support}$ for the s largest indices in $\left(x^{k}\right)$
$x^{k}=\arg \min \left\{\left\|y-A_{\text {real }}^{c s} x\right\|_{2}, \operatorname{supp}(\mathrm{x}) \subseteq \mathrm{T}\right\}$
$r^{k}=y-A_{\text {real }}^{c s} x^{k}$
End for if $\left(\left\|r^{k}\right\|_{2} \leq\right.$ EPS $)$
Output: $\boldsymbol{x}=x^{k}$

\section{Simulation Analysis}

By using Monte Carlo simulation for repetition 500 times randomly to test the performance of modified recovery algorithms and compare it with conventional ones. Simulations are divided into two parts as follows. 


\section{A. Simulation Parameters}

Using sinusoidal signal of dimensions $(n, 1)$ where $n=1024$, the input signal is variability in the time domain in the range of $t=0: n-1$ which is described by [14],

$$
\begin{aligned}
& x=0.6 \sin 2 \pi(29 / n) t-1.5 \sin 2 \pi(100 / n) t+\sin 2 \pi(200 / n) t+0.8 \sin 2 \\
& \pi(400 / n) t+2 \sin 2 \pi(500 / n) t-\sin 2 \pi(600 / n) t
\end{aligned}
$$

- Converting this signal to sparse signal $x_{f}$ in the frequency domain by using fast Fourier transform with sparsity level $\mathrm{k}=6$. Choosing the measurement matrix $A$ orthogonal and Gaussian random matrix. Normalized measures for the measurement length to the signal length are defined by $R=m / n$ which $R$ varies in the range of [0.1:0.9] where the measurements length $\mathrm{m}=\operatorname{round}\left(\mathrm{R}^{*} \mathrm{n}\right)$. UsingL1-NORM, COSAMP, HTP and IHT as an optimization technique that is used for achieving the optimal solution [9].The performance of the proposed algorithms will be tested by using the following matrices:

Average Normalized Mean Square Error:

$$
\mathrm{ANMSE}=\frac{1}{500} \sum_{1}^{500}\left[\|x-\hat{x}\|_{2}^{2} /\|x\|_{2}^{2}\right],
$$

Average Normalized Signal to Error ratio (ANSER) in dB:

$$
\text { ANSER }=\frac{1}{500} \sum_{1}^{500}\left[10 \log _{10} p s / p e\right]=\frac{1}{500} \sum_{1}^{500}\left[10 \log _{10} \frac{y^{*} y^{\prime}}{m} / \frac{\text { res } * \text { res }}{m}\right]
$$

Where, the res $=y-A_{\text {real }}^{c s} \hat{x}_{f}$ and iteration time in the range of change of $\mathrm{R}$.

\section{B. Simulation Results and Discussion}

The simulation results are divided into four parts, all parts provide a comparison between the conventional algorithms and the proposed ones with respect to different evaluation matrices as described in the figures. Fig. 4 shows the variation of the compression ratio $\mathrm{m} / \mathrm{n}$ that represents the measurement vector length $\mathrm{m}$ normalized to the signal length $\mathrm{n}$ with respect to the average run time. This measurement length must be less than the signal length as possible for minimum error in the compressed sensing system. Increasing the compression ratio $(\mathrm{m} / \mathrm{n})$ will produce increasing atan iteration time because of using a number of measurements must be needed more processing time. 


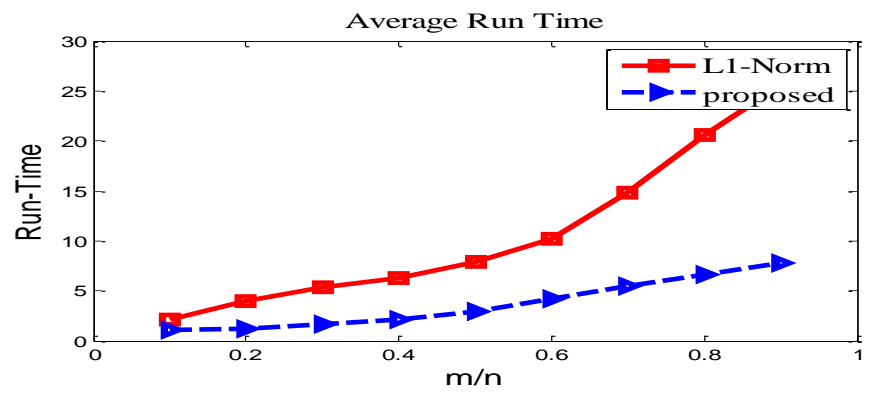

(a) L1-norm algorithm

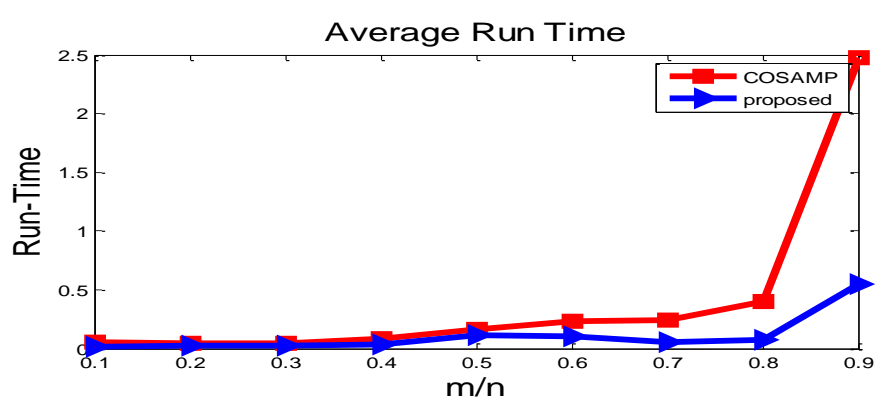

(b) COSAMP algorithm

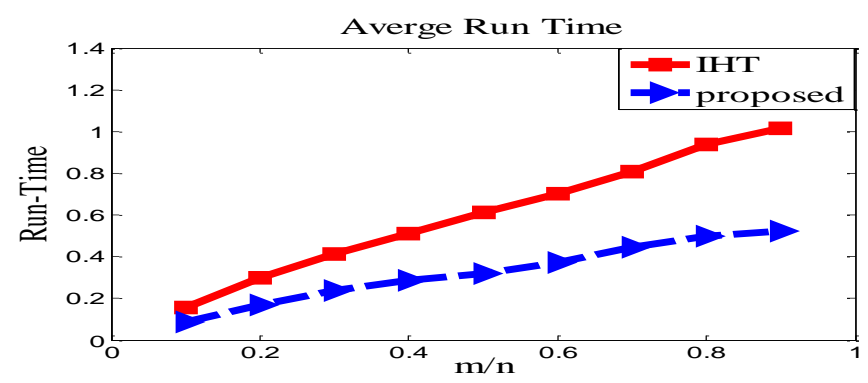

(c) IHT algorithm

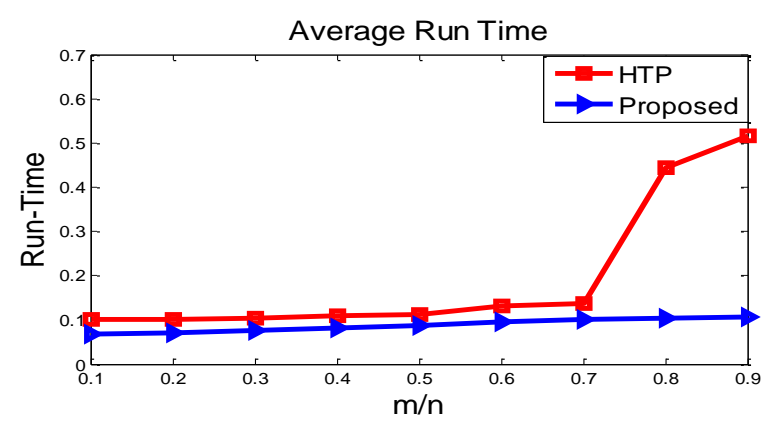

(d) HTP algorithm

Fig 4: Comparison between the proposed algorithms and the conventional ones with respect to the average run time. 
This figure will compare between all of L1-NORM, COSAMP, IHT and HTP algorithms with the proposed ones.Taking any point of compression ratio in Fig.4 (a) at $(m / n)=0.8$, The run time of conventional L1-NORM will be reduced by using the proposed algorithm from 22 to 7 . The proposed algorithm decreases the run time because of the complexity due to dealing with the imaginary parts will take a larger time than using real parts even if we doubled the size of the matrix it will take less time than using imaginary parts. Another reason for reducing the runtime with using the proposed algorithm is that we need the sensing matrix as input for the recovery algorithm, therefore using the real matrix is faster in dealing and saving the time than dealing with the imaginary matrix. Fig.4 (b) provide a comparison between conventional COSAMP and the proposed one. At any point of $m / n=0.9$, improvement will appear in the proposed algorithm by 5 times than conventional algorithm. Using an IHT algorithm in Fig.4 (c) which the proposed algorithm also provides enhancement at the run time by percentage 50\%. In the end, using proposed HTP in Fig.4 (c) reduces the run time also than conventional algorithm.

Figure 5 will illustrate the performance of the four proposed algorithms and compared it with the conventional algorithms with respect to the average mean square error. The mean square error decreases with increasing the compression ratio because of increasing measurements mean that more samples represent the signal. Whenever the measurements near to the signal length, the error is decreasing. Fig.5 (a) provides a comparison between the proposed and the conventional L1-NORM where the error due to using the proposed algorithm appears as fixed line but it decreases gradually in the range of $10^{-7}$. The proposed algorithm recovers the sparse signal with minimum error than the conventional one because the proposed algorithm removes the complexity and dealing with the real matrix which contribute to minimize the error than dealing with complex matrix. Fig.5 (b) shows a comparison between the performance of COSAMP and the proposed algorithm where the proposed algorithm provides enhancement in the error reduction by percentage 50\%. Fig.5 (c) clarifies that the proposed algorithm decreases the error than the conventional IHT which provide enhancements in the compressed sensing system. Fig.5 (d) represents the clear improvement in the error due to using the modified HTP than the conventional one where the error is close to zero. 


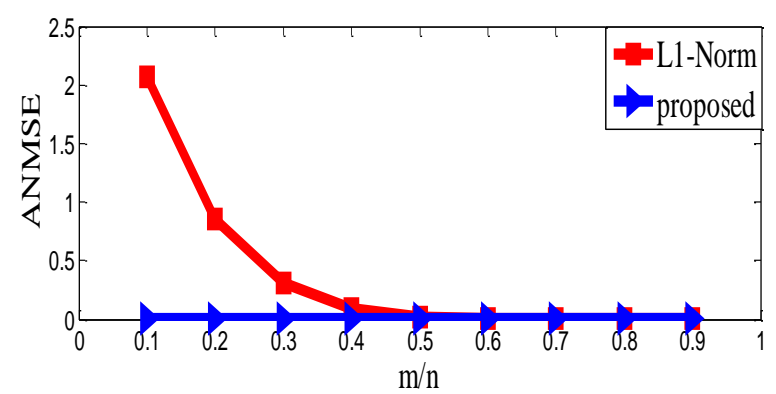

(a) L1-norm algorithm

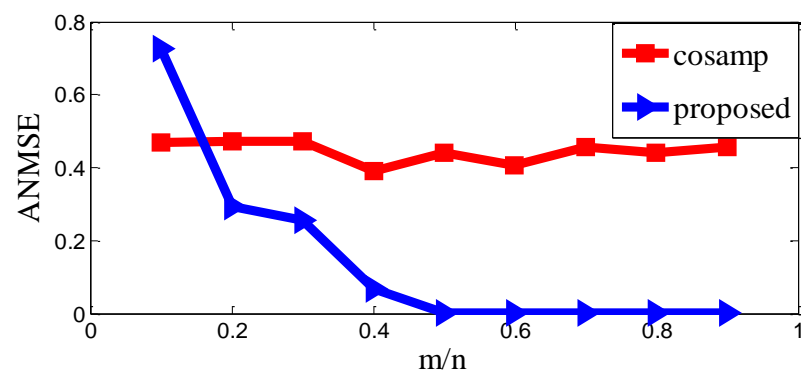

(b) COSAMP algorithm

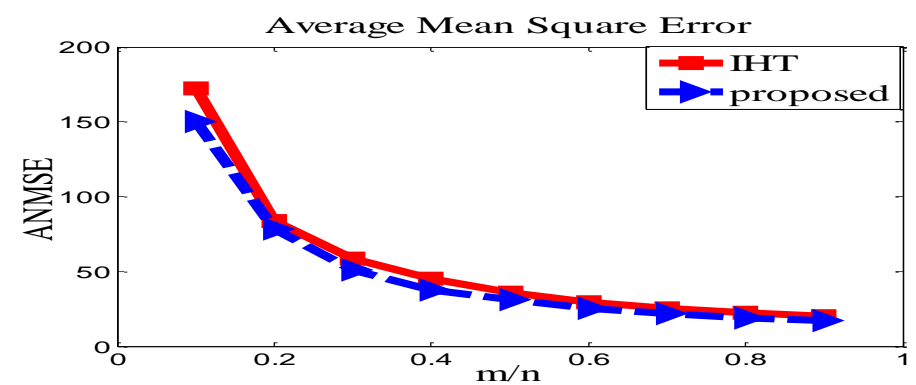

(c) IHT algorithm

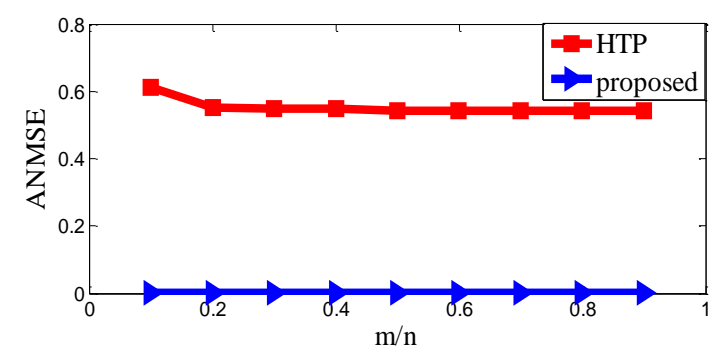

(d) HTP algorithm

Fig 5: Comparison between the proposed algorithms and the conventional ones with respect to the average mean square 
Fig.6 studies the variations between the compression ratio $(\mathrm{m} / \mathrm{n})$ and the average signal to error ratio. It illustrates the response of applying the complex to real transformation of different algorithms which providesimprovement in signal to error ratio in all cases. As the number of measurements increase to near to Nyquist rate, the recovery enhanced which the signal to error ratio increases. Fig.6 (a)describes that the signal to error ratio will be increased with increasing the compression ratio, but when using the proposed algorithm, the signal to error ratio increases than using the conventional L1-NORM. Fig.6 (b) shows that by using COSAMP algorithm the signal to ratio also increases with increasing the ratio $(\mathrm{m} / \mathrm{n})$ but it is noticeable that the signal to error ratio improves when applying the proposed technique by pecentage $50 \%$ approximately. Fig.6(c) compares between the conventional IHT and the proposed one where the signal to error ratio improves by using the proposed algorithm.Fig.6 (d) the signal to error ratio appears as fixed line but the modified HTP changes increasing in the range of $260 \mathrm{~dB}$ and the conventional HTP changed in the range of $20 \mathrm{~dB}$ where the proposed provide higher improvement than conventional algorithm

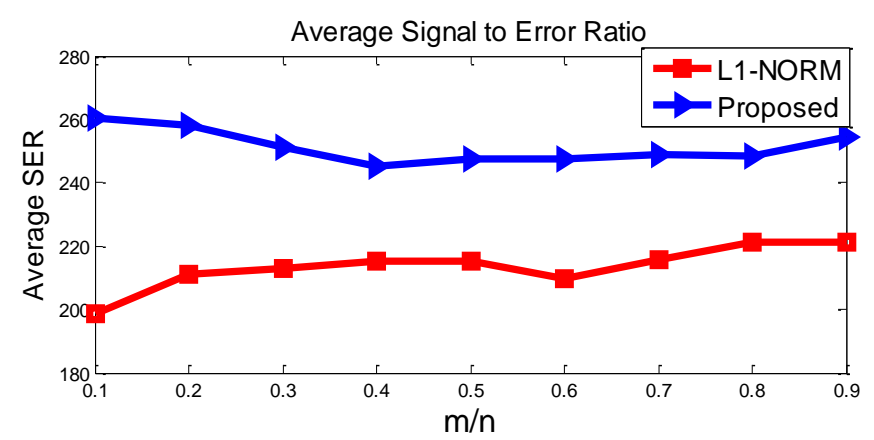

(a) L1-norm algorithm

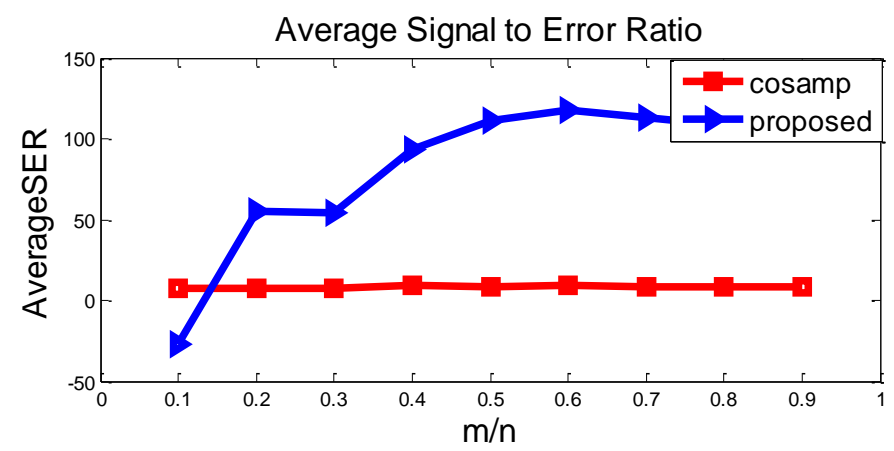

(b) COSAMP algorithm 


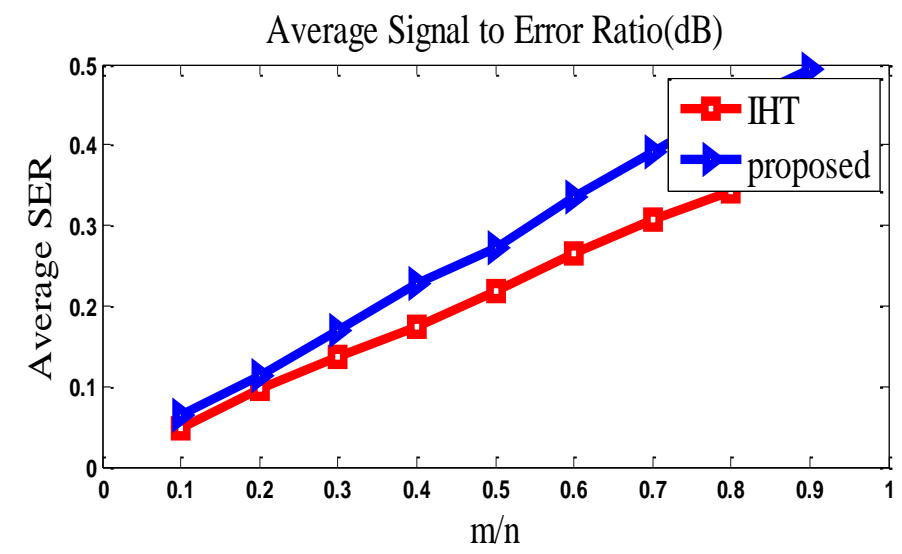

(c) IHT algorithm

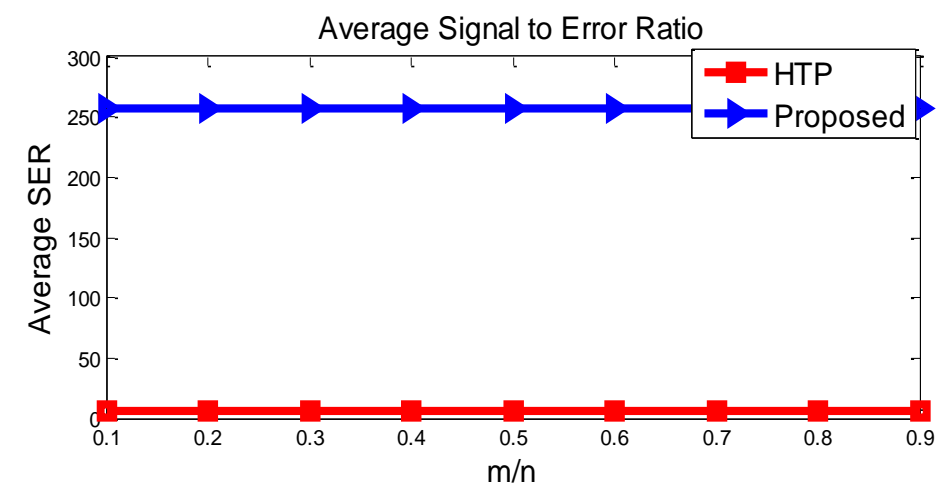

(d) HTP algorithm

Fig 6. Comparison between the proposed algorithms and the conventional ones with respect to the average Signal to Error ratio.

\section{Conclusions}

The important process in compressed sensing is the recovery process that recover the sparse signal from the minimum number of measurements by using different optimization algorithms in minimum time and minimum error and high signal to error ratio. This paper introduced modification in all recovery algorithms that provide improvements for the performance of recovery algorithms when compared to the conventional ones. Compressed sensing system performance improved by using the proposed algorithm. Converting the signal that is not completely sparse in the time domain to sparse in the frequency domain by using Fourier transform to 
be allowed to use in the compressed sensing system will produce complexity in both the measurement matrix and the sparse signal. Removing the complexity by using the complex to a real transformation algorithm which introduces enhancement in the performances of recovery algorithms inthe compressed sensing system.

\section{References}

[1] D. L. Dohoho,"Compressed sensing," IEEE Trans-Inf. Theory, Vol. 52, PP.1289-1306, 2006.

[2] E. J. Candies,"The restricted isometry properties and its implications for compressed sensing,"C. R. Math. Acad. Sci. Paris, Vol.346, No.9, PP.589-592, May2008.

[3] S. P. Boyd and L. Vandenberghe," Convex optimization," Cambridge Univ Pr, March 2004.

[4] E.Candes and T.Ta., "Decoding by linear programming," IEEE Trans. on Inf. Theory, Vol.40698, PP.1-22, 2005.

[5] J. A. Tropp and A. C. Gilbert," Signal recovery from random measurements via orthogonal matching pursuit," IEEE Trans. Info. Theory, PP. 4655-4666, 2007.

[6] Needell D., Tropp JA., "COsaMP: Iterative signal recovery for incomplete and inaccurate sample,"Applied and Computational Harmonic Analysis. Elsevier J., PP. 301-321, 2008.

[7] T. Blvmensath and M. Davies, "Iterative hard thresholding for compressed sensing,"Applied and Computational Harmonic Analysis. Elsevier J., Vol.27, No.3, PP.265-274, 2009.

[8] Somon Foucart,"Hard Thresholding Pursuit: An algorithm for compressive sensing," SIAM J.Numer.Anal, Vol.49, No.6, PP.25432563, 2011.

[9] Kazunori Hayashi, Nasaaki Nagahara and Toshiyuki Tanaka,"A user's guide to compressed sensing for communication system, "IEICE Trans. Comm. Vol.E96-1B, No.3, 2013.

[10] Needell D., Tropp J., Vershynin R., "Greedy signal recovery review," Signals, Systems and Computers, 2nd Asilomar Conference, PP.10481050, October 2008.

[11] Zaixing HE., Takahiro OGAWA., and Miki Haseyama," The simplest measurement matrix for compressed sensing of natural images," IEEE 17th International Conference on Image Processing, Hong Kong, September 2010. 
[12] Emmanuel J. Candès and Michael B. Wakin "An introduction to compressive sampling," IEEE Signal Processing Magazine, Vol. 25, PP. 21 - 30, March 2008.

[13] Wafaa A.Shalaby,Waleed saad, Mona Shokair, and Moawad Dessouky, "An Efficient Recovery Algorithms using Complex to Real Transformation of compressed Sensing," 33rd National, Radio Science Conference (NRSC), 2016.

[14] Lei Hu, Zhiguang Shi., Jianxiong Zhou, and Qiang Fu., " Compressed sensing of complex sinusoids: an approach based on dictionary refinement," IEEE Trans. On Signal Processing, Vol. 60, No. 7, July 2012. 


\section{ملخص البحث باللغة العربية}

هذا البحث يقدم تحسين لأداء الانظمه المستخدمه في استرجاع الاشـاره الاصليه

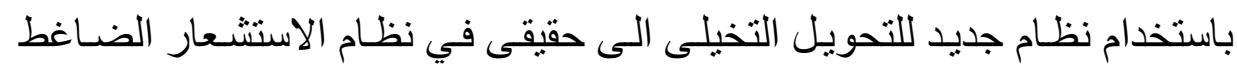

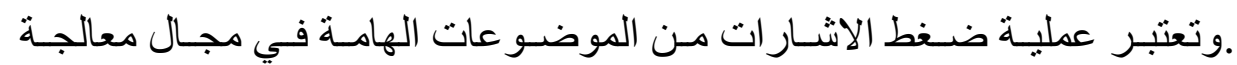

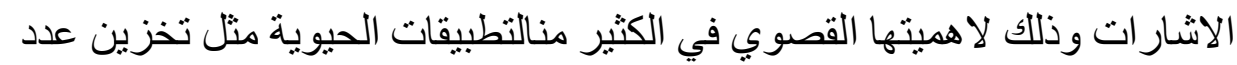

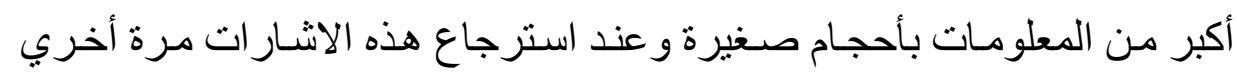

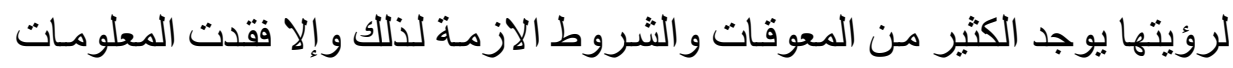

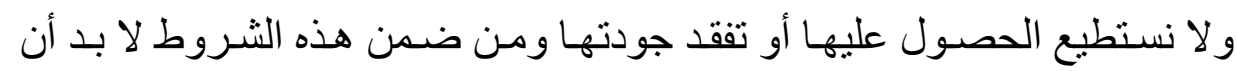

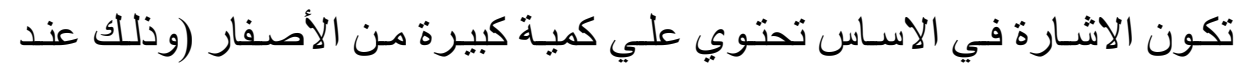

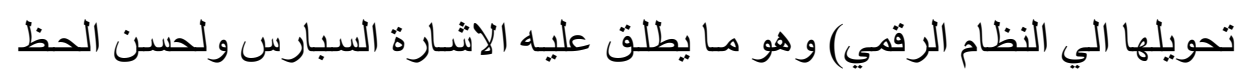

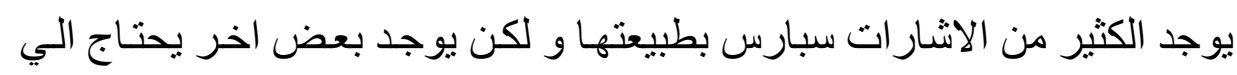

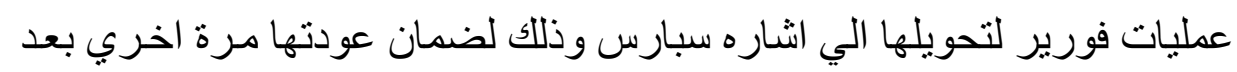

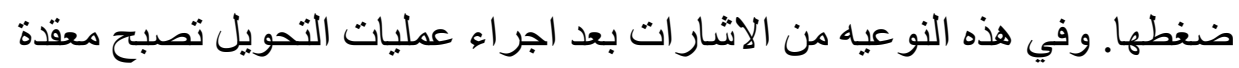

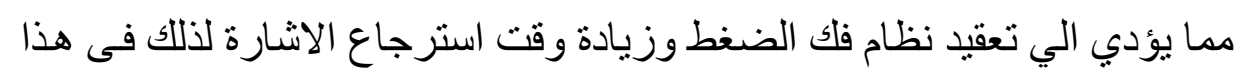

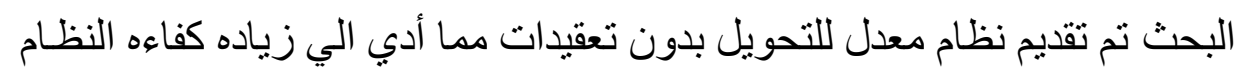

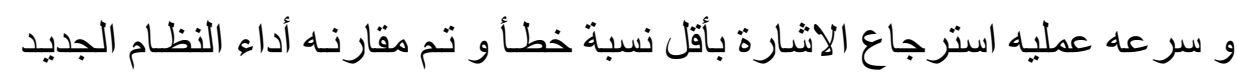

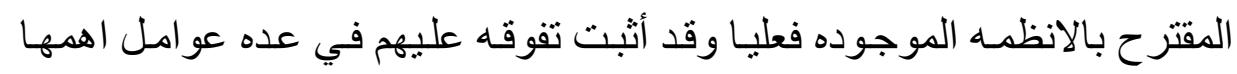

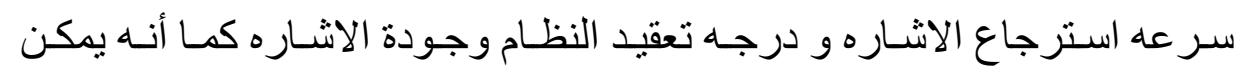
إستخدام هذه التقنيه المقترحة لتعديل أي من الانظمة المتاحة حاليا لتحسن أداءها. 